\title{
Readmission and mortality in patients $\geq 70$ years with acute myocardial infarction or heart failure in the Netherlands: a retrospective cohort study of incidences and changes in risk factors over time
}

\author{
P. Jepma $(\mathbb{D})$ G. ter Riet · M. van Rijn · C. H. M. Latour · R. J. G. Peters · W. J. M. Scholte op Reimer · B. M. Buurman
}

Published online: 4 February 2019

(c) The Author(s) 2019

\begin{abstract}
Objectives To determine the risk of first unplanned allcause readmission and mortality of patients $\geq 70$ years with acute myocardial infarction (AMI) or heart failure (HF) and to explore which effects of baseline risk factors vary over time.

Methods A retrospective cohort study was performed on hospital and mortality data (2008) from Statistics Netherlands including 5,175 (AMI) and 9,837 (HF) patients. We calculated cumulative weekly incidences for first unplanned all-cause readmission and mortality during 6 months post-discharge and explored patient characteristics associated with these events. Results At 6 months, 20.4\% and 9.9\% (AMI) and 24.6\% and $22.4 \%$ (HF) of patients had been readmitted or had died, respectively. The highest incidences were found in week 1 . An increased risk for 14-day mortal-
\end{abstract}

Electronic supplementary material The online version of this article (https://doi.org/10.1007/s12471-019-1227-4) contains supplementary material, which is available to authorized users.

P. Jepma $(\bowtie)$ C C. H. M. Latour · W. J. M. Scholte op Reimer · B. M. Buurman

ACHIEVE Centre for Applied Research, Faculty of Health, Amsterdam University of Applied Sciences, Amsterdam, The Netherlands

p.jepma@hva.nl

\section{G. ter Riet}

Amsterdam UMC, Department of General Practice,

University of Amsterdam, Amsterdam, The Netherlands

M. van Rijn · B. M. Buurman

Amsterdam UMC, Department of Internal Medicine, Section of Geriatric Medicine, University of Amsterdam, Amsterdam, The Netherlands

\section{R. J. G. Peters · W. J. M. Scholte op Reimer}

Amsterdam UMC, Department of Cardiology, University of Amsterdam, Amsterdam, The Netherlands ity after AMI was observed in patients who lived alone (hazard ratio (HR) 1.57, 95\% confidence interval (CI) 1.01-2.44) and within 30 and 42 days in patients with a Charlson Comorbidity Index $\geq 3$. In HF patients, increased risks for readmissions within 7, 30 and 42 days were found for a Charlson Comorbidity Index $\geq 3$ and within 42 days for patients with an admission in the previous 6 months (HR 1.42, 95\% CI 1.12-1.80). Nonnative Dutch HF patients had an increased risk of 14day mortality (HR 1.74, 95\% CI 1.09-2.78).

Conclusion The risk of unplanned readmission and mortality in older AMI and HF patients was highest in the 1st week post-discharge, and the effect of some risk factors changed over time. Transitional care interventions need to be provided as soon as possible to prevent early readmission and mortality.

Keywords Acute myocardial infarction - Heart failure · Mortality · Patient readmission · Transitional care

\section{What's new?}

- The highest incidences of readmission and mortality in older patients with acute myocardial infarction (AMI) or heart failure (HF) were found in week 1 . The timing of transitional care interventions needs to be improved to prevent early events.

- Patients with comorbidities, an admission in the previous 6 months, patients living alone and non-native Dutch patients were at highest risk for early readmission and mortality.

- Better alignment of transitional interventions is needed, as risk factors and timing of readmission and mortality differ for older AMI and HF patients. Therefore, disease-oriented treatments in transitional interventions might be integrated to reduce risks. 


\section{Introduction}

Older patients who have been recently discharged after hospital admission for cardiac events are at high risk of readmission and mortality [1]. Research has shown that factors such as higher age [2, 3], the presence of comorbidities [4,5], being single [2], low socioeconomic status $[2,6]$ and an admission in the previous 6 months [7] increase the risk of readmission and mortality.

Transitional care interventions (TCIs) aim to improve continuity of care after discharge through multidisciplinary collaboration, structured discharge planning and early follow-up home visits and have proven to lower the risk of readmission and mortality [8-10]. The start and duration of TCIs vary. Le Berre et al. [10] found that TCIs started after an average of 7.9 days (SD 6.2) post-discharge and lasted for an average of 179.7 days (SD 158.5), showing large diversity in duration of interventions. It is currently unclear what the optimal time window is for TCIs in (various subgroups of) older cardiac patients. Better delineation of which older cardiac patients would benefit most in which time windows would allow the most efficient deployment of TCIs.

Therefore, we determined the risk of a first unplanned all-cause readmission and mortality of patients $\geq 70$ years with acute myocardial infarction (AMI) or heart failure (HF) and explored the extent to which effects of particular baseline risk factors vary over time.

\section{Methods}

\section{Data sources}

We used the National Medical Registration (LMR) of 2008 (and 2009 for the follow-up) from Statistics Netherlands [11] in which $88 \%$ of all hospital admissions in the Netherlands were registered anonymously. The LMR was linked to the Dutch Population Registry (GBA), which contains demographic characteristics. Record linkage was successful in $88.9 \%$ of hospital-admitted patients [11]. The dates of death were obtained from the Causes of Mortality registry. The Integrated Income Data of Household registry (IIDH) was used to retrieve additional information about residence, living circumstances and annual income.

\section{Study population}

Patients with an unplanned hospital admission in 2008 were included. Eligible patients were identified with help of the International Classification of Diseases, 9th revision, Clinical Modification (ICD-9-CM). Patients were eligible if they were $\geq 70$ years old, had a discharge diagnosis of AMI (ICD-9 410) or HF (ICD9 428) and had a length of stay $\geq 1$ day. The first car- diac admission that met these criteria was considered as the index admission. Transfers to other hospitals or wards during this admission were taken as part of the same admission. No approval of the Medical Ethics Committee was necessary as data were used from national registries with anonymous information.

\section{Outcomes and risk factors}

We examined the cumulative weekly incidence of a first unplanned all-cause readmission and mortality within 6 months. We identified potential risk factors at baseline and examined the extent to which their associations with the outcomes varied over time. An unplanned all-cause readmission was defined as any non-elective admission occurring at least 1 day after discharge from the index admission in any hospital. Risk factors were selected based on availability in the LMR, GBA and IIDH registries (Electronic Supplementary Material: Table S1-S4).

\section{Statistical analysis}

We described data using counts and percentages for categorical variables and means with standard deviations (SD) or medians with interquartile ranges (IQR) as appropriate.

We calculated the cumulative weekly incidence for unplanned all-cause readmission and mortality in AMI and HF patients until 6 months post-discharge. The number of events per week post-discharge was divided by the number of persons at risk at the start of that week. Follow-up ended if patients experienced the event of interest, died (in case of readmission) or at 6 months after the index admission if a target event did not occur.

Then, we examined by extended multivariable Cox regression analyses [12] to what extent the effects of baseline risk factors on unplanned all-cause readmission and mortality until 6 months post-discharge varied across five time points: 3, 7, 14, 30 and 42 days post-discharge. This modified Cox regression analysis is a time-to-event analysis to study if the association of a particular factor with the outcome varies over time. It involves risk factor-time interaction terms into the regression models (dummy variables for time were coded $0=$ early and $1=$ late) [12]. To reduce the number of statistical tests, we performed chunk tests comparing models with and without all risk factortime interaction terms based on the likelihood ratio test. Statistically non-significant chunk tests $(p \geq 0.05)$ were interpreted as an indication that the extended model including the interaction terms did not lead to a better fit and standard multivariable Cox regression analysis was preferred. We took statistically significant chunk tests as an indication that the model with interactions fitted the data better. We performed a stepwise backward procedure with a $p$-value for entry and removal of 0.05 and 0.10 , respectively. Statistically sig- 
nificant risk factor-time interactions were interpreted as risk factors whose effect varied over time. We expressed all hazard ratios (HRs) such that values greater than 1 indicate higher risk at the earlier time point. HRs were displayed on a logarithmic scale to enhance compact visualisation of scattered estimates (Figs. 2, 3 and 4). Analyses were performed with SPSS Statistics 22.0 (SPSS Inc., Chicago, IL, USA).

\section{Results}

A total of 15,012 patients $\geq 70$ years had an unplanned hospital admission and discharge diagnosis of AMI $(n=5,175 ; 35.5 \%)$ or HF $(n=9,837 ; 65.5 \%)$. During the index admission, 1,878 patients (12.5\%) died: $576 \mathrm{AMI}$ patients (11.1\%) and 1,302 HF patients (13.2\%). Thus, a total of 13,134 patients discharged with a diagnosis of AMI $(n=4,599)$ or HF $(n=8,535)$ were included. Tab. 1 shows the patient characteristics.

\section{Cumulative incidence of a first unplanned all-cause readmission}

Fig. 1a shows the cumulative incidences of a first unplanned all-cause readmission within 6 months postdischarge. In total, $20.4 \%$ of AMI patients $(n=937)$ and $24.6 \%$ of HF patients $(n=2,103)$ had been readmitted. The highest incidences were found in week 1 : 4.8\% (AMI) and 3.7\% (HF) were readmitted, respec- tively. After week 3, the cumulative weekly incidences were lower than $2 \%$.

\section{Cumulative incidence of mortality}

Fig. 1b shows the cumulative incidences of mortality within 6 months post-discharge. In total, 9.9\% of AMI patients $(n=457)$ and $22.4 \%$ of HF patients $(n=1,914)$ had died. The highest cumulative incidences were found in week 1: $1.4 \%$ (AMI) and $2.1 \%$ (HF) died, respectively. After week 1, the cumulative incidence of mortality in AMI patients was lower than $1 \%$. In HF patients, a more gradual decline in cumulative incidence was found with incidences between $1.5 \%$ and $0.5 \%$ from week 4 onward.

\section{Risk factors of a first unplanned all-cause readmission}

In AMI patients, the associations between risk factors and readmission did not vary over time. Therefore, the analyses resulted in the same model for all time windows (Table S1).

In $\mathrm{HF}$ patients, a higher Charlson Comorbidity Index (CCI) increased the risk of early readmission within 7,30 and 42 days, e.g. patients with a CCI $\geq 3$ had a $56 \%$ greater risk of readmission within 7 days (HR 1.56, 95\% confidence interval (CI) 1.15-2.11) than patients with a CCI of 1 (reference category). Women had a $24 \%$ lower risk of readmission within 7 days
Table 1 Baseline characteristics of included patients

\begin{tabular}{|c|c|c|}
\hline & $\begin{array}{l}\text { Acute myocardial infarction } \\
(n=4,599)\end{array}$ & $\begin{array}{l}\text { Heart failure } \\
(n=8,535)\end{array}$ \\
\hline Male, $n(\%)$ & $2,464(53.6 \%)$ & $3,749(43.9 \%)$ \\
\hline Age, mean (SD) & $79.2(6.0)$ & $81.8(6.3)$ \\
\hline Native Dutch, $n(\%)$ & $4,123(89.6 \%)$ & $7,620(89.3 \%)$ \\
\hline Patients living alone ${ }^{\mathrm{b}}, n(\%)$ & $1,999(43.5 \%)$ & $4,607(54.0 \%)$ \\
\hline Living in an institution, $n$ (\%) & $314(6.8 \%)$ & $1,189(13.9 \%)$ \\
\hline Length of stay (days), median (IQR) & $6.0(4.0-10.0)$ & $7.0(5.0-12.0)$ \\
\hline Admission in the previous 6 months, $n(\%)$ & $158(3.4 \%)$ & $846(9.9 \%)$ \\
\hline \multicolumn{3}{|l|}{$C C^{\beta}[28], n(\%)$} \\
\hline - Score 1 & $2,933(63.8 \%)$ & $5,379(63.0 \%)$ \\
\hline - Score 2 & $1,200(26.1 \%)$ & $1,897(22.2 \%)$ \\
\hline - Score $\geq 3$ & $466(10.1 \%)$ & $1,259(14.8 \%)$ \\
\hline \multicolumn{3}{|l|}{ Annual income ${ }^{\complement}, n(\%)$} \\
\hline$-\leq € 16,801$ & $2,538(55.2 \%)$ & 4,026 (47.2\%) \\
\hline$->€ 16,801$ & $2,059(44.8 \%)$ & $4,509(52.8 \%)$ \\
\hline \multicolumn{3}{|l|}{ Type of hospital, $n(\%)$} \\
\hline - General hospital & $1,874(40.7 \%)$ & $4,482(52.5 \%)$ \\
\hline - Tertiary referral hospital & $2,469(53.7 \%)$ & $4,776(44.2 \%)$ \\
\hline - University hospital & $256(5.6 \%)$ & $277(3.2 \%)$ \\
\hline \multicolumn{3}{|c|}{$\begin{array}{l}\text { IQR interquartile range, } N \text { number, } S D \text { standard deviation } \\
\text { aCharlson Comorbidity Index (CCI) [28]: a weighted index to classify comorbid conditions based on their } 1 \text {-year mortality } \\
\text { prognosis. The index was categorised as above. A CCI of } 1 \text { was the reference category, because acute myocardial } \\
\text { infarction and heart failure both score } 1 \text { point in the original CCI } \\
\text { bPatients living alone or with children } \leq 18 \text { years old } \\
\text { 'Dichotomised, based on median income in the dataset }\end{array}$} \\
\hline
\end{tabular}


Fig. 1 a The incidence rates of a first unplanned allcause readmission within 6 months. b The incidence rates of mortality within tive incidence was calcuand deaths by the number of patients at risk for each week until 6 months post-discharge) 6 months (The cumulalated for each week postdischarge by dividing the number of readmissions

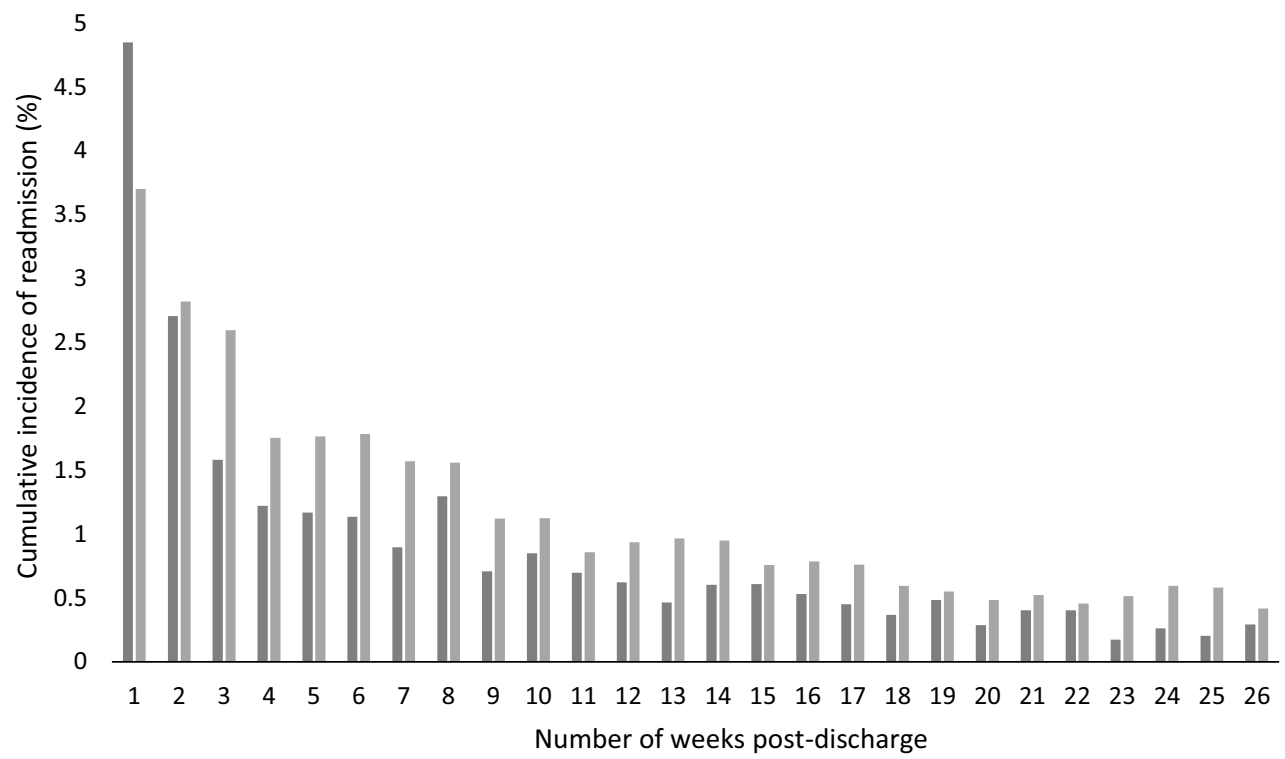

a Acute myocardial infarction Heart failure

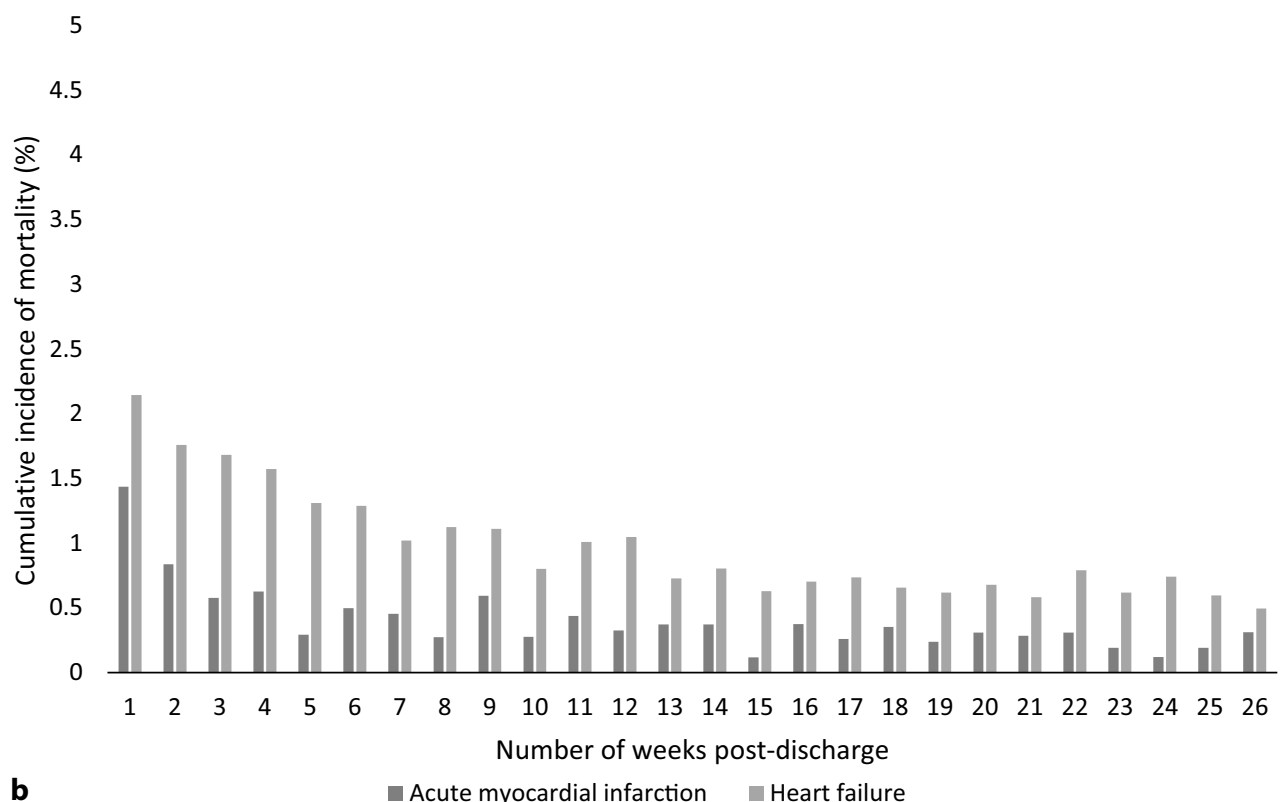

(HR 0.76, 95\% CI 0.60-0.97) than men. Patients with an admission in the previous 6 months before the index hospitalisation had no greater risk of readmission within 30 days (HR 1.23, 95\% CI 0.97-1.57) than those without such previous admissions, while a $42 \%$ greater risk was found for readmission within 42 days (HR 1.42, 95\% CI 1.12-1.80) (Fig. 2, Table S2).

\section{Risk factors for mortality}

Fig. 3 (and Table S3) shows the extended Cox regression analyses of early mortality post-discharge in AMI patients. Patients living alone had a $57 \%$ greater risk of mortality within 14 days (HR 1.57, 95\% CI 1.01-2.44). Patients with a CCI $\geq 3$ had a $121 \%$ greater risk of mor- tality within 42 days (HR 2.21, 95\% CI 1.22-4.02) than those with a CCI of 1 .

In HF patients, risk factor-time interactions were found for early mortality in all time windows (Fig. 4, Table S4). The risk factor-time interaction for readmission indicated an increased risk of mortality in all time windows. Non-native Dutch patients, compared to native Dutch, had a $74 \%$ greater risk of early mortality within 14 days (HR 1.74, 95\% CI 1.09-2.78). A 15\% lower risk of early mortality within 42 days was found for every 10 years of age (HR $0.85,95 \%$ CI $0.72-0.99$ ). Lower risks of early mortality were also found for patients living in an institution or with an admission in the previous 6 months. 


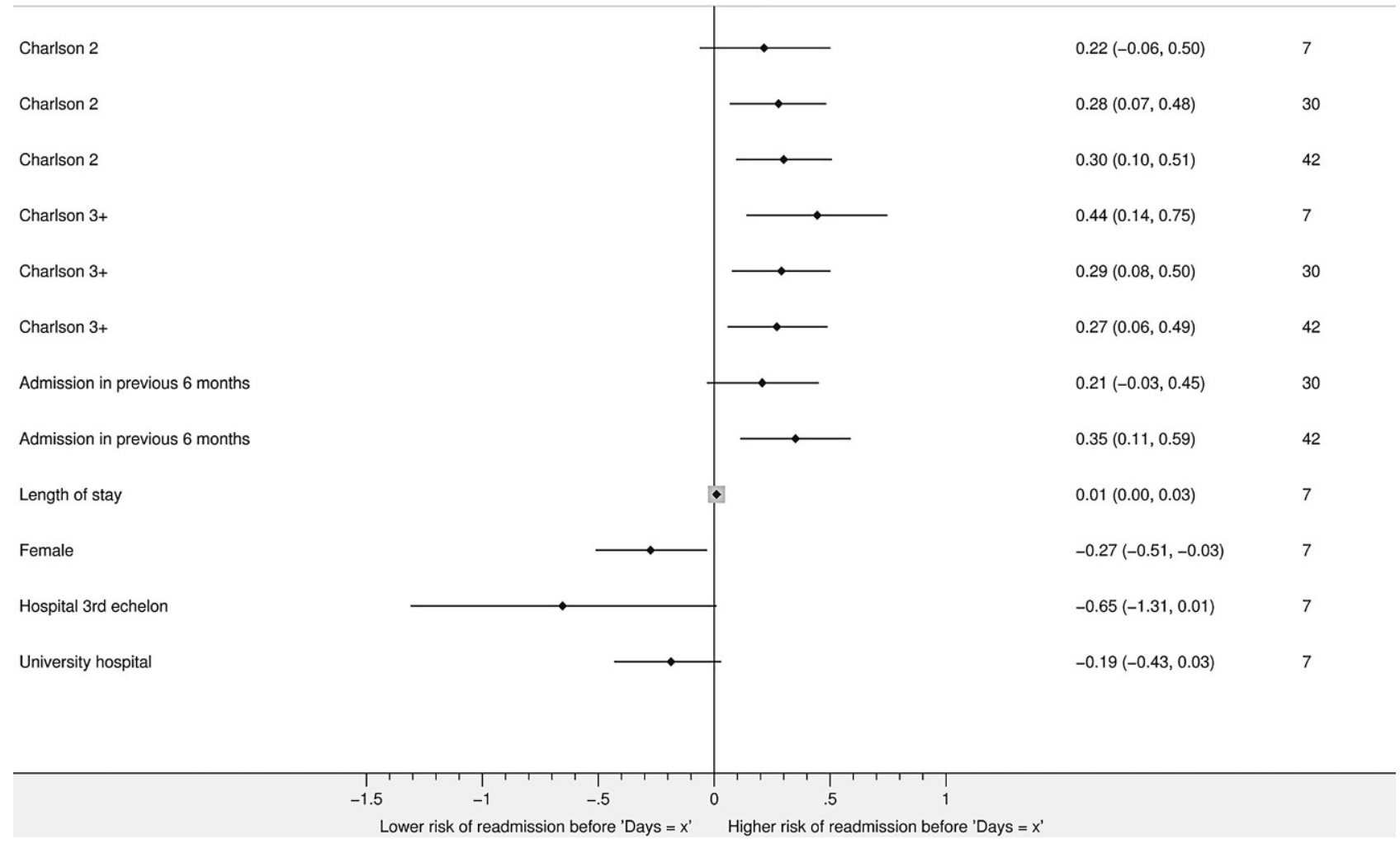

Fig. 2 Risk factors for a first unplanned all-cause readmission whose effects change over time in heart failure patients (Hazard ratios (HRs) are displayed on a logarithmic scale to en- hance compact visualisation of scattered estimates. The exact HRs are shown in Table S2, Charlson 2 Charlson Comorbidity Index of 2, Charlson 3+ Charlson Comorbidity Index of $\geq 3$ )

\section{Discussion}

In this retrospective cohort study of older cardiac patients after an unplanned hospital admission in the Netherlands, we found that 20.4\% (AMI) and $24.6 \%$ (HF) had an unplanned all-cause readmission and 9.9\% (AMI) and 22.4\% (HF) had died within 6 months post-discharge. The highest incidences were found in the 1st week post-discharge. Patients with comorbidities, an admission in the previous 6 months, patients living alone and non-native Dutch patients were at highest risk of early readmission and mortality.

Consistent with the literature from the United States [13-15], this study on older Dutch cardiac patients confirms that the highest readmission and mortality rates were found right after discharge and that risks were higher and prolonged in HF patients compared to AMI patients [15]. These results suggest that the needs of older cardiac patients are insufficiently fulfilled in the early period post-discharge. The average start of TCIs after 8 days post-discharge [10] might already be too late to have a preventive effect on early readmission and mortality. Therefore, the timing of TCIs may need improvement.
We found that higher CCIs increased the risk of early readmission (HF) and mortality (AMI) at several time points. During hospital admission, older cardiac patients mainly receive disease-oriented treatments based on disease-specific guidelines, which are in turn based on studies that commonly exclude older and multimorbid patients [16, 17]. However, older cardiac patients often suffer from multiple comorbidities including diabetes, chronic pulmonary disease and renal failure [4, 5, 18]. Donzé et al. [19] found that the focus on acute illness during admission may lead to insufficient monitoring of comorbidities and increase the risk of exacerbations post-discharge. A broader assessment of older cardiac patients' needs during hospital admission might be required [20].

\section{Strengths and limitations}

One of the strengths of our study is that we used a large nationwide database and had the opportunity to link and combine hospital and sociodemographic data with 1-year follow-up. This resulted in fairly rich data to examine risk factors for readmission and mortality. To our knowledge, our study is the first to examine change in those effects over time. While $11 \%$ of the 


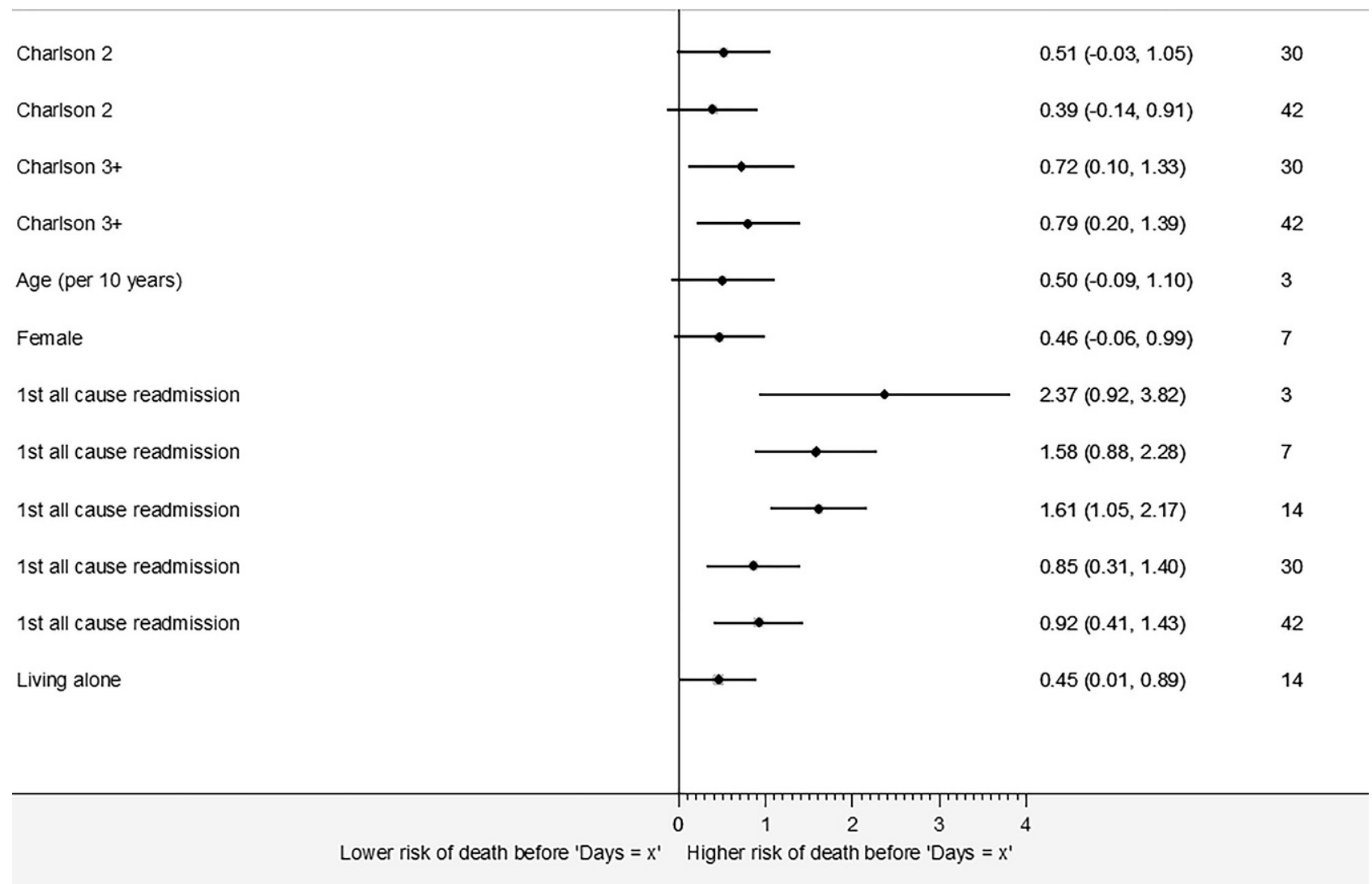

Fig. 3 Risk factors for mortality whose effects change over time in acute myocardial infarction patients (HRs are displayed on a logarithmic scale to enhance compact visualisation of scattered estimates. The exact HRs are shown in Ta-

cases were excluded because no linkage between hospital and sociodemographic data was possible, previous research from Statistics Netherlands showed that the number of linkable admissions were reliable for statistical analyses [21].

This study also has limitations. First, we had access only to the registries of Statistics Netherlands in 2008 and 2009 for the follow-up. Due to national trends, the incidences of readmission and mortality might nowadays have increased in HF patients and decreased in AMI patients [22]. Although the incidences might be different, we expect that the highest incidences are still found in the 1st week post-discharge. Second, the LMR contained only administrative data which precluded adjustment for cardiovascular and geriatric risk factors that are known to increase the risk of readmission and mortality (e.g. history of cardiovascular disease, disability and polypharmacy). Third, we were unable to adjust for competing risk in patients that had died before experiencing an unplanned all-cause readmission which might have resulted in an underestimation for readmission [23]. Finally, the CCIs in our data may be too low because of the underreporting of ble S3, Charlson 2 Charlson Comorbidity Index of 2, Charlson $3+$ Charlson Comorbidity Index of $\geq 3$ All-cause readmission. This covariate indicates the first all-cause readmission after the index admission)

comorbidities in medical files. This may have caused an underestimation of the effect on readmission and mortality.

\section{Implications of findings}

Hospitalised high-risk older cardiac patients need to be identified as soon as possible to guide them during care transitions. Instead of single disease-oriented treatments, a broad view on older cardiac patients' needs is necessary [20]. Around discharge, adequate communication between hospital and community care providers, e.g. accurate and timely discharge letters, and continuity of care after discharge have proven to reduce readmissions [24]. In addition, careful assessment of patients' readiness for discharge might be needed, as some high-risk patients might even be discharged before stable recovery [25].

While single disease-oriented interventions during hospital admission are not suitable in older cardiac patients [16, 17, 19], disease management interventions might be integrated in TCIs. More disease-specific guidance after discharge, e.g. symptom monitor- 


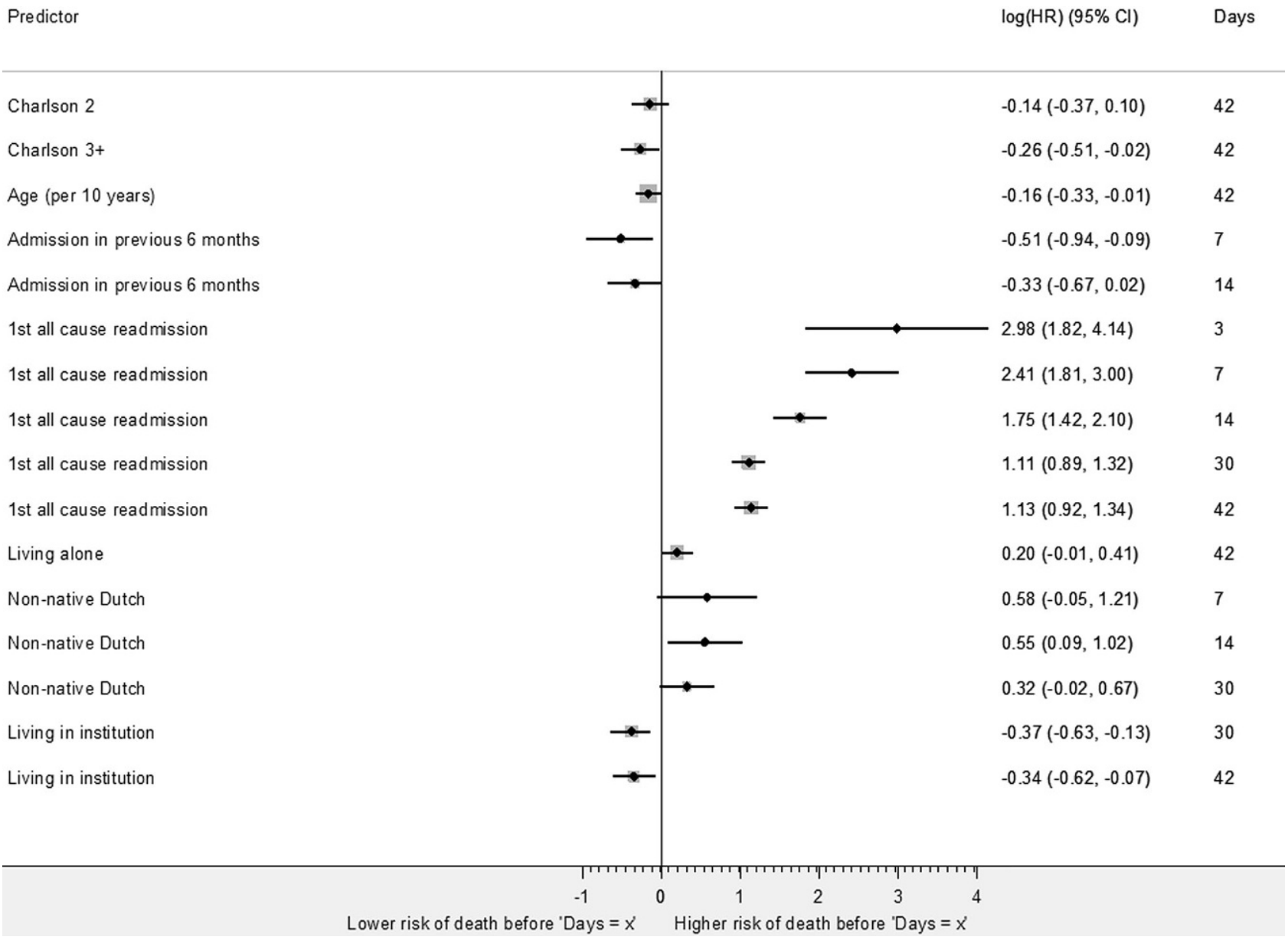

Fig. 4 Risk factors for mortality whose effects change over time in older heart failure patients (HRs are displayed on a logarithmic scale to enhance compact visualisation of scattered estimates. The exact HRs are shown in Table S4, Charl-

ing, medication reconciliation and specific guidance in medication and lifestyle adherence, might also help to reduce the risk of readmission and mortality [8, 26]. Personalised interventions might be required as HF patients were at higher and prolonged risk compared to AMI patients, and risk factors for readmission and mortality changed over time. Although readmission diagnoses are heterogeneous, early detection and proactive interventions might limit complications [13, 27].

\section{Conclusion}

The incidences of unplanned all-cause readmission and mortality in older AMI and HF patients were highest in the 1st week post-discharge, and the effects of several risk factors for these events at discharge changed over time. Transitional care interventions need to be provided as soon as possible in admitted high-risk older patients with AMI or HF to prevent early readmission and mortality. son 2 Charlson Comorbidity Index of 2, Charlson 3+ Charlson Comorbidity Index of $\geq 3$ All-cause readmission. This covariate indicates the first all-cause readmission after the index admission)

Acknowledgements The authors thank Statistics Netherlands and Dutch Hospital Data for providing the data.

Funding This work was supported by the Netherlands Organization for Health Research and Development (ZonMw) as part of the 'From knowledge to Action II program' (grant number 520002002) and by the Netherlands Organisation for Scientific Research (NWO) (grant number 023.009.036) to P. Jepma.

Conflict of interest P. Jepma, G. ter Riet, M. van Rijn, C.H.M. Latour, R.J.G. Peters, W.J.M. Scholte op Reimer and B.M. Buurman declare that they have no competing interests.

Open Access This article is distributed under the terms of the Creative Commons Attribution 4.0 International License (http://creativecommons.org/licenses/by/4.0/), which permits unrestricted use, distribution, and reproduction in any medium, provided you give appropriate credit to the original author(s) and the source, provide a link to the Creative Commons license, and indicate if changes were made. 


\section{References}

1. Krumholz HM, Lin Z, Keenan PS, et al. Relationship between hospital readmission and mortality rates for patients hospitalized with acute myocardial infarction, heartfailure, or pneumonia. JAMA. 2013;309(6):587-93.

2. Calvillo-King L, Arnold D, Eubank KJ, et al. Impact of social factors on risk of readmission or mortality in pneumonia and heart failure: systematic review. J Gen Intern Med. 2013;28(2):269-82.

3. Huynh QL, Saito M, Blizzard CL, et al. Roles of nonclinical and clinical data in prediction of 30-day rehospitalization or death among heart failure patients. J Card Fail. 2015;21(5):374-81.

4. Rushton CA, Satchithananda DK, Jones PW, et al. Noncardiovascular comorbidity, severity and prognosis in nonselected heart failure populations: a systematic review and meta-analysis. Int J Cardiol. 2015;196:98-106.

5. Lee CS, Chien CV, Bidwell JT, et al. Comorbidity profiles and inpatient outcomes during hospitalization for heart failure: an analysis of the U.S. nationwide inpatient sample. BMC Cardiovasc Disord. 2014;14:73. https://doi.org/10.1186/ 1471-2261-14-73.

6. Perk J, De Backer G, Gohlke H, et al. European guidelines on cardiovascular disease prevention in clinical practice (version 2012). The fifth joint task force of the European Society of Cardiology and other societies on cardiovascular disease prevention in clinical practice (constituted by representatives of nine societies and by invited experts). Eur HeartJ.2012;33(13):1635-701.

7. Mudge AM, Kasper K, Clair A, et al. Recurrent readmissions in medical patients: a prospective study. J Hosp Med. 2011;6(2):61-7.

8. Albert NM, Barnason S, Deswal A, et al. Transitions of care in heart failure: a scientific statement from the American Heart Association. Circ Heart Fail. 2015;8(2):384-409.

9. Feltner C, Jones CD, Cene CW, et al. Transitional care interventions to prevent readmissions for persons with heart failure: a systematic review and meta-analysis. Ann Intern Med. 2014;160(11):774-84.

10. LeBerreM,Maimon G, Sourial N, etal. Impact oftransitional care services for chronically ill older patients: a systematic evidence review. JAm Geriatr Soc. 2017;65(7):1597-608.

11. Statistics Netherlands. Documentation report Dutch medical registration. Den Haag: Statistics Netherlands; 2010.

12. Kleinbaum DG, Klein M. Extension of the Cox proportional hazards model for time-dependent variables. Survival analysis: a self-learning text. New York: Springer; 2005. pp. 211-56.

13. Ranasinghe I, Wang Y, Dharmarajan K, et al. Readmissions after hospitalization for heart failure, acute myocardial infarction, or pneumonia among young and middleaged adults: a retrospective observational cohort study. Plos Med. 2014;11(9):e1001737. https://doi.org/10.1371/ journal.pmed.1001737.t008.
14. Dharmarajan K, Hsieh AF, Lin Z, et al. Diagnoses and timing of 30-day readmissions after hospitalization for heart failure, acute myocardial infarction, or pneumonia. JAMA. 2013;309(4):355-63.

15. Dharmarajan K, Hsieh AF, Kulkarni VT, et al. Trajectories of risk after hospitalization for heart failure, acute myocardial infarction, or pneumonia: retrospective cohort study. BMJ. 2015;350:h411. https://www.bmj.com/content/350/bmj. h411.

16. Forman DE, Rich MW, Alexander KP, et al. Cardiac care for older adults. Time for a new paradigm. J Am Coll Cardiol. 2011;57(18):1801-10.

17. Tinetti M, Naik A, Dodson J. Moving from disease-centered to patient goals-directed care for patients with multiple chronic conditions. JAMA Cardiol. 2016;1(1):9-10.

18. Buddeke J, Bots ML, van Dis I, et al. Trends in comorbidity in patients hospitalised for cardiovascular disease. Int J Cardiol. 2017;248:382-8.

19. Donzé J, Lipsitz S, Bates DW, et al. Causes and patterns of readmissions in patients with common comorbidities: retrospective cohort study. BMJ. 2013;347:f7171. https:// www.bmj.com/content/347/bmj.f7171.

20. Bell SP, Orr NM, Dodson JA, et al. What to expect from the evolving field of geriatric cardiology. J Am Coll Cardiol. 2015;66(11):1286-99.

21. deBruinA, Kardaun J, GastF, etal. Record linkage of hospital discharge register with population register: experiences at Statistics Netherlands. Stat J UN Econ Comm Eur. 2004;21:23-32.

22. Leening MJ, Siregar S, Vaartjes I, et al. Heart disease in the Netherlands: a quantitative update. Neth Heart J. 2014;22(1):3-10.

23. Austin PC, LeeDS, FineJP. Introduction to the analysis of survival data in the presence of competing risks. Circulation. 2016;133(6):601-9.

24. Hesselink G, Schoonhoven L, Barach P, et al. Improving patient handovers from hospital to primary care: a systematic review. Ann Intern Med. 2012;157(6):417-28.

25. Verhaegh KJ, Jepma P, Geerlings SE, et al. Not feeling ready to go home: a qualitative analysis of chronically ill patients' perceptions on care transitions. Int J Qual Health Care. 2018; https://doi.org/10.1093/intqhc/mzy139.

26. Snaterse M, Dobber J, Jepma P, et al. Effective components of nurse-coordinated care to prevent recurrent coronary events: a systematic review and meta-analysis. Heart. 2016;102(1):50-6.

27. Dharmarajan K, Krumholz HM. Strategies to reduce 30day readmissions in older patients hospitalized with heart failure and acute myocardial infarction. Curr Geriatr Rep. 2014;3(4):306-15.

28. Charlson ME, Pompei P, Ales KL, et al. A new method of classifying prognostic comorbidity in longitudinal studies: development and validation. J Chronic Dis. 1987;40(5):373-83. 